Revista de Psicología Vol. 29 (2), 2011 (ISSN 0254-9247)

\title{
Representaciones estereotípicas y expresión del prejuicio en el Perú: la mirada desde la pobreza
}

\author{
Gina Pancorbo ${ }^{1}$, Agustín Espinosa ${ }^{2}$ y Rosa María Cueto ${ }^{3}$ \\ Pontificia Universidad Católica del Perú
}

\begin{abstract}
Se estudian estereotipos y prejuicios hacia distintos grupos étnicos que presentan los habitantes de una zona de pobreza extrema en el Callao, Perú. Se desarrolló un diseño mixto de investigación con una fase cuantitativa donde se aplicaron encuestas $(n=115)$ y una cualitativa donde se realizaron 4 grupos focales $(\mathrm{n}=30)$. Los resultados muestran representaciones estereotípicas que comprenden grupos de alto (blancos) y bajo estatus (andinos, amazónicos y afroperuanos). Los grupos evaluados son representados a través de contenidos estereotipos ambivalentes. Los blancos son percibidos como instrumentales, corruptos y poco patriotas, mientras que los de bajo estatus como poco instrumentales pero cálidos y simpáticos. Comparando estos resultados con los obtenidos por Espinosa, Calderón, Güimac \& Burga (2007), se observa que las representaciones estereotípicas de los participantes de sectores pobres son estructuralmente similares a las de personas de sectores medios. Sin embargo, el prejuicio asignado a grupos de alto estatus resulta mayor en la muestra de pobreza extrema, ya que se atribuye a sus miembros prácticas discriminatorias y excluyentes que afectan a los grupos de bajo estatus.

Palabras clave: identidad social, estereotipos, prejuicio, racismo.
\end{abstract}

Stereotyping representations and prejudice expression in Peru: The vision from poverty Stereotypes and prejudices towards different ethnic groups held by people from an extremely impoverished area in Callao, Peru were studied. A mixed methodology was designed, comprised of a survey study $(n=115)$ and four focus groups $(n=30)$. Results show the existence of specific stereotypes to represent groups of high (Whites) and low status (Andeans, Amazonians and Afro-Peruvians). Every assessed group was described through ambivalent stereotyping contents. Results show that white people were perceived as instrumentalist, corrupts and non-patriotic while low status groups were seen as low in instrumentalism but warmth and sympathetic. Comparing these results with Espinosa, Calderon, Güimac and Burga (2007), it becomes evident that stereotyping representations held by people of poor strata are structurally similar to those held by people of middle-class groups. However, prejudice toward high status groups' members is stronger among poor respondents when compared with middle class respondents, because impoverished people are likely to consider members of high status groups as individuals with discriminatory and excluding behaviors against people of low status groups.

Keywords: Social identity, stereotypes, prejudice, racism. 

Los estereotipos son los ejemplos más estudiados de la categorización social (Tajfel \& Forgas, 1981), y se definen como un conjunto de creencias interrelacionadas de los miembros de un grupo que describen las percepciones acerca de determinados miembros de otro grupo (McGarty, Yzerbyt \& Spears, 2002).

Stangor (2000) refiere que los estereotipos tienen fundamento en la realidad y eso los hace de gran utilidad en términos de una aproximación cognitiva al medio social. La estereotipia es funcional para la adaptación al entorno y no debe ser vista como un proceso cognitivo inferior o socialmente destructivo (Moghaddam, Taylor \& Wright, 1993). Sin embargo, en ocasiones, el proceso puede derivar en generalizaciones sin fundamento que desvalorizan a determinados grupos sociales y derivan en conflicto intergrupal (Moghaddam, 1998).

Dada su funcionalidad, los estereotipos permiten racionalizar y justificar la forma cómo se dan las diferencias en una estructura social donde existen tanto grupos dominantes como dominados, y aceptar las condiciones sociales, económicas y políticas que estructuran dicha sociedad (Yzerbyt, Rocher \& Schadron, 1997).

Los estereotipos vienen comúnmente acompañados del prejuicio, el cual se define como una actitud negativa hacia un grupo social o hacia un individuo, considerando su pertenencia a dicho grupo (Allport, 1954; Dovidio, 2001; Dovidio \& Gaertner, 1999; Stangor, 2000).

1 Licenciada en Psicología Social por la PUCP. Ha sido asistente de docencia en el Departamento de Psicología de la PUCP. Contacto: Avenida Universitaria 1801, Lima 32, Perú; gpancorbo@pucp.pe

2 Doctor en Psicología Social por la Universidad del País Vasco, España. Profesor auxiliar del Departamento de Psicología de la PUCP. Contacto: Avenida Universitaria 1801, Lima 32, Perú; agustin.espinosa@pucp.edu.pe

3 Magister en Psicología Comunitaria por la Universidad de Chile. Profesora auxiliar del Departamento de Psicología de la PUCP. Contacto: Avenida Universitaria 1801, Lima 32, Perú; rcueto@pucp.pe 
Un tipo especial de prejuicio es el racismo, el cual añade a la definición del prejuicio, la superioridad de la propia raza y además, racionaliza prácticas institucionales y expresiones de cultura que formalizan la dominación jerárquica de un grupo sobre otro (Jones, como se citó en Zárate, 2009).

Existe evidencia de que la división de las razas es socialmente construida, antes que una consecuencia de diferencias biológicas, lo que se plasma en la forma cómo opera la categorización racial; por ejemplo a partir de la observación del fenotipo o de la apariencia general de una persona (Fiske \& Taylor, 2007).

Con relación a ello, un problema importante al que se enfrenta la investigación en el tema es que la existencia del racismo usualmente pasa desapercibido. Lo anterior se expresa en el concepto de racismo aversivo, por el cual las personas desarrollan, a través de la socialización, creencias y sentimientos de los que tienen poca conciencia o que tratan de disociar de la imagen no-prejuiciosa que desean mostrar. Las emociones asociadas al prejuicio de los racistas aversivos son la ansiedad, la incomodidad, el disgusto y el miedo, como consecuencia de una evaluación positiva hacia el endogrupo y negativa hacia el exogrupo (Gaertner \& Dovidio, 1986).

Debido a que los racistas aversivos tienen valores igualitarios, no discriminan directamente en formas que podrían ser atribuidas al racismo clásico; en vez de ello, discriminan de forma poco intencional y cuando su comportamiento puede ser justificado sobre la base de algún factor que no sea racial (economía, capacidad, inteligencia, etc.) (Dovidio, 2001).

En el caso peruano, el racismo implica que las personas tiendan a valorar negativamente lo andino, negando aquella parte de su identidad que los relaciona con lo indígena y se sobre-identifiquen con lo blanco y lo occidental (Bustamante, 1986; Portocarrero, 1992). A su vez, conlleva que los grupos de bajo estatus sientan envidia, odio y resentimiento por los de alto estatus, y que estos últimos sientan desprecio, miedo y culpa con respecto a los de bajo estatus (Portocarrero, 1992). 
En un estudio realizado por Espinosa et al. (2007), se identificó la forma en que personas de niveles socioeconómicos medios y medioaltos de Lima Metropolitana valoraban más a categorías sociales cuyas representaciones estereotípicas las posicionaban en una situación de alto estatus, como a los peruanos blancos, a los descendientes de asiáticos y a los mestizos, antes que a grupos asociados a categorías sociales de bajo estatus, como los peruanos de origen andino, amazónico y los afroperuanos.

En dicho estudio se encontró estereotipos ambivalentes asociados al grupo de peruanos blancos tales como el desarrollo, la capacidad y el éxito, y otros asociados al individualismo, la corrupción y la falta de patriotismo. Del mismo modo, se encontró ambivalencia en los estereotipos de los peruanos andinos, amazónicos y afroperuanos, ya que se les asoció con conformismo, atraso, subdesarrollo e incapacidad, pero también con valentía, patriotismo y solidaridad.

$\mathrm{Al}$ respecto, no es extraño que en la sociedad peruana tanto el poder y el logro, como la corrupción percibida como un medio para alcanzarlos, sean características atribuidas a los peruanos blancos. Por el contrario, los andinos, amazónicos y afroperuanos son asociados a un conjunto de características que los representan como alejados del poder (Espinosa, 2011; Espinosa et al., 2007).

El presente estudio busca describir y analizar los estereotipos y prejuicios hacia grupos étnicos peruanos que presentan los pobladores de una zona urbano-marginal del Callao en Perú. Adicionalmente, se propone comparar tales expresiones estereotípicas y de prejuicio con las encontradas por Espinosa et al. (2007) en un estudio con una muestra de clase media de Lima Metropolitana.

También se busca analizar la relación de la identificación con el Perú con la expresión de la identificación con grupos étnicos y los prejuicios étnicos, así como la relación entre la expresión del prejuicio individual y el prejuicio percibido en los participantes del estudio. Por último se plantea describir en profundidad las razones y argumentos que subyacen a la asignación de atributos estereotípicos a los distintos grupos evaluados en la presente investigación. 


\section{Método}

\section{Participantes}

Los participantes del estudio fueron pobladores de asentamientos humanos del distrito de Ventanilla, Provincia Constitucional del Callao, Perú. Tomando en cuenta la ubicación de los asentamientos y las características de las viviendas visitadas, se puede establecer que los participantes pertenecen a sectores sociales que viven en condiciones de marginalidad urbana y extrema pobreza (APEIM, 2010).

La muestra de la etapa cuantitativa de la investigación fue de 115 personas, cuyas edades fluctuaron entre 19 y 54 ańos de edad, con una media de 31.4 ańos $(D E=9.24)$ y una mediana de 30 años; el $53 \%$ de la muestra fueron mujeres y el $47 \%$ hombres.

La gran mayoría de los participantes no nació en Lima, sino en otros departamentos de la costa (23.5\%), de la sierra $(39.1 \%)$ y de la selva (16.5\%). El $11.3 \%$ de la muestra afirmó tener como lengua materna el quechua, mientras que el $87.8 \%$ reportó el español como primera lengua.

Por su parte, el $81.7 \%$ de la muestra logró como máximo culminar la secundaria o algún nivel educativo inferior y el 18.3\% restante alcanzó a cursar estudios superiores, la mayoría de los cuales quedaron incompletos.

En la segunda etapa de la investigación, de carácter cualitativo, se convocó a 30 personas a cuatro grupos focales, cuyas características se detallan en la tabla 1.

\section{Tabla 1}

Distribución de participantes en los cuatro grupos focales

\begin{tabular}{cccc}
\hline Grupo Focal & Hombres & Mujeres & Total \\
\hline 1 & 6 & 3 & 9 \\
2 & 0 & 8 & 8 \\
3 & 1 & 8 & 9 \\
4 & 2 & 2 & 4 \\
\hline Total & 9 & 21 & 30 \\
\hline
\end{tabular}




\section{Instrumentos}

Ficha de datos control

En la etapa cuantitativa se usó una ficha de control que indagó acerca de los siguientes datos demográficos: sexo, edad, año de nacimiento, lugar de nacimiento, lengua materna, segunda lengua, último año de estudios y ocupación.

Escala de estereotipos (Espinosa, 2003)

La escala consiste en una lista de 23 adjetivos identificados por Espinosa (2003) en un estudio sobre identidad nacional. Se presentaron los adjetivos a los participantes y se pidió que marquen los que mejor consideran que caracterizan a cada uno de los siguientes grupos sociales: peruanos en general, peruanos mestizos, peruanos de origen andino, peruanos de origen amazónico, peruanos de origen asiático, afroperuanos y peruanos blancos. En la presente investigación, se añadieron tres atributos más a los considerados por el autor citado: clase alta, clase media y clase baja.

Escala de nivel de identificación étnica y valoración de los grupos sociales (Espinosa et al., 2007)

La escala consta de dos partes:

1. Identificación con grupos sociales: para medir la identificación se utilizó el siguiente enunciado: Sobre los grupos de peruanos que he mencionado, ¿Cuál es su grado de identificación con los peruanos blancos/mestizos/negros/ de origen amazónico/ de origen andinol de origen asiático? Luego de ello, se presentaron las categorías sociales antes referidas, a fin de que los participantes marquen el nivel de identificación que tienen con cada una ellas en una escala del 1 al 5 , donde $1=$ nada y $5=$ totalmente. Posteriormente, se pidió a los participantes que, tomando en cuenta su aspecto físico y el de sus familiares, elijan la categoría que mejor los describiera, de las seis que se les presentaron (peruanos mestizos, peruanos negros, 
peruanos blancos, peruanos de origen andino, peruanos de origen amazónico, peruanos de origen asiático).

2. Valoración de los grupos sociales: la valoración de los grupos étnicos se realizó a partir de las respuestas al siguiente enunciado: ¿Cuál es la valoración que usted hace de los siguientes grupos étnicos y nacionales? Luego se presentó a los participantes la lista de categorías sociales consideradas en la investigación, ante la cual respondieron en una escala del 1 al 10, donde 0 = muy mala y 10 = muy buena. Posteriormente, se preguntó ¿Cuál cree usted que es la valoración que tienen los otros peruanos de los siguientes grupos teniendo en cuenta que 0 es "muy mala" y 10 "muy buena"?, luego de lo cual se presentó nuevamente la lista de categorías y las alternativas de respuesta antes descritas.

Guia de discusión acerca de estereotipos y prejuicios hacia grupos sociales

La Guía de Grupo Focal fue elaborada especialmente para el presente estudio y tuvo como objetivos conocer a profundidad las razones que subyacen a la estereotipia de los grupos étnicos considerados, validar los resultados cuantitativos de la investigación en cuanto a los atributos asignados a los grupos, y conocer los estereotipos y atribuciones con respecto a las relaciones intergrupales en el Perú.

\section{Procedimiento}

En la etapa cuantitativa de la investigación se consultó a los participantes si querían responder al cuestionario, para lo cual se les pidió leer un consentimiento informado. Una vez que aceptaban participar, se les aplicó de manera asistida un cuestionario con las escalas mencionadas en el acápite anterior. Posteriormente, se codificaron los cuestionarios y se descargaron en una base de datos en el paquete estadístico SPSS 15. Luego, se realizaron los análisis estadísticos descriptivos, de correspondencias, de correlaciones y regresión lineal. Luego, se unieron las bases de datos del presente estudio y del estudio de Espinosa et al. (2007) a 
fin de realizar análisis descriptivos y de comparación de medias para muestras independientes.

Para la etapa cualitativa, se revisaron los resultados obtenidos en la primera etapa de la investigación que sirvieron para la construcción de la Guía de Grupo Focal.

A fin de realizar los grupos focales, se contactó a participantes con características demográficas similares a los participantes de la etapa cuantitativa y se les consultó acerca de su disponibilidad para participar en el estudio y su consentimiento para la grabación de la sesión grupal. Las sesiones grupales fueron dirigidas por uno de los investigadores.

Una vez realizados los grupos focales, se transcribió la información recogida y se realizó el análisis de contenido de la misma.

Los resultados de ambas partes de la investigación se usaron en la producción de resultados generales: los resultados cualitativos sirvieron para dar profundidad a los resultados cuantitativos, complementarlos y ejemplificarlos a través de citas textuales. Lo anterior permitió la elaboración de la discusión y conclusiones de acuerdo con los objetivos de la investigación.

\section{Resultados}

\section{Estereotipos hacia los peruanos en general y hacia grupos sociales evaluados}

Con relación a los estereotipos de los peruanos en general y de los grupos étnicos asociados por los participantes del presente estudio, se presenta en la tabla 2 los cinco atributos más elegidos por los participantes. 


\section{Tabla 2}

Atributos estereotipicos más importantes de los grupos sociales evaluados, en porcentajes

\begin{tabular}{|c|c|c|c|c|c|c|}
\hline $\begin{array}{l}\text { Peruanos } \\
\text { en general }\end{array}$ & Andinos & $\begin{array}{c}\text { Amazó- } \\
\text { nicos }\end{array}$ & Asiáticos & $\begin{array}{c}\text { Afrope- } \\
\text { ruanos }\end{array}$ & Blancos & Mestizos \\
\hline $\begin{array}{c}\text { Trabaja- } \\
\text { dores } \\
(80.0)\end{array}$ & $\begin{array}{c}\text { Trabaja- } \\
\text { dores } \\
(74.8)\end{array}$ & $\begin{array}{c}\text { Trabaja- } \\
\text { dores } \\
(78.3)\end{array}$ & $\begin{array}{c}\text { Trabaja- } \\
\text { dores } \\
(80.0)\end{array}$ & $\begin{array}{c}\text { Trabaja- } \\
\text { dores } \\
(88.7)\end{array}$ & $\begin{array}{c}\text { Trabaja- } \\
\text { dores } \\
(77.4)\end{array}$ & $\begin{array}{c}\text { Exitosos } \\
(57.4)\end{array}$ \\
\hline $\begin{array}{c}\text { Alegres } \\
(65.2)\end{array}$ & $\begin{array}{c}\text { Alegres } \\
(73.9)\end{array}$ & $\begin{array}{c}\text { Alegres } \\
(67.8)\end{array}$ & $\begin{array}{c}\text { Valientes } \\
(65.2)\end{array}$ & $\begin{array}{c}\text { Valientes } \\
(67.0)\end{array}$ & $\begin{array}{c}\text { Desarro- } \\
\text { llados } \\
(63.5)\end{array}$ & $\begin{array}{c}\text { Clase alta } \\
(57.4)\end{array}$ \\
\hline $\begin{array}{c}\text { Patriotas } \\
(61.7)\end{array}$ & $\begin{array}{c}\text { Valientes } \\
(62.6)\end{array}$ & $\begin{array}{c}\text { Honrados } \\
\quad(61.7)\end{array}$ & $\begin{array}{c}\text { Solidarios } \\
(62.6)\end{array}$ & $\begin{array}{c}\text { Honrados } \\
(67.0)\end{array}$ & $\begin{array}{c}\text { Exitosos } \\
(60.9)\end{array}$ & $\begin{array}{c}\text { Capaces } \\
(50.4)\end{array}$ \\
\hline $\begin{array}{l}\text { Solidarios } \\
\qquad(58.3)\end{array}$ & $\begin{array}{c}\text { Patriotas } \\
\quad(55.7)\end{array}$ & $\begin{array}{c}\text { Patriotas } \\
\text { (61.7) }\end{array}$ & $\begin{array}{c}\text { Patriotas } \\
\text { (60.9) }\end{array}$ & $\begin{array}{l}\text { Alegres } \\
(60.9)\end{array}$ & $\begin{array}{c}\text { Capaces } \\
(56.5)\end{array}$ & $\begin{array}{c}\text { Desarro- } \\
\text { llados } \\
(49.6)\end{array}$ \\
\hline $\begin{array}{c}\text { Confor- } \\
\text { mistas } \\
(53.9)\end{array}$ & $\begin{array}{c}\text { Solidarios } \\
\text { (52.2) }\end{array}$ & $\begin{array}{c}\text { Solidarios } \\
\text { (60.9) }\end{array}$ & $\begin{array}{c}\text { Capaces } \\
(57.4)\end{array}$ & $\begin{array}{c}\text { Patriotas } \\
(60.9)\end{array}$ & $\begin{array}{c}\text { Cumplidos } \\
\text { (52.2) }\end{array}$ & $\begin{array}{c}\text { Alegres } \\
(49.6)\end{array}$ \\
\hline
\end{tabular}

Por otro lado, se encontró la relación entre las categorías socioétnicas y los 26 atributos considerados en la presente investigación, a través de un análisis de correspondencias cuyo modelo fue significativo $\left(\chi^{2}=546.578, p<.001\right)$ y que explicó el $69.0 \%$ de la inercia total.

La figura 1 ubica a los peruanos en general cercanos a atributos como ociosos, atrasados, incumplidos, conformistas y mentirosos. Más abajo se ubican los andinos y asiáticos considerados como patriotas, alegres, solidarios, incapaces, de clase media y fracasados. También relacionados a estos atributos y un poco más lejos de los grupos mencionados se ubican los amazónicos, relacionados a características como trabajadores, valientes, capaces, cumplidos, honestos y de confianza. Comparten estas dos últimas características los afroperuanos, a quienes también se les considera honrados. El atributo de clase baja se encuen- 
tra, por su parte, relacionado a las categorías de afroperuanos, andinos y asiáticos en mayor medida que a los amazónicos. Por otro lado, la categoría mestizos se asocia a atributos como individualistas y no quieren a su patria, y en menor medida, a atributos como corruptos, mentirosos, no confiables y exitosos. Por último, cercano al grupo de peruanos mestizos, se ubica la categoría de blancos, quienes son considerados como exitosos y desarrollados, así como individualistas, capaces, no confiables y de clase alta, aunque en menor medida que los dos primeros atributos mencionados.

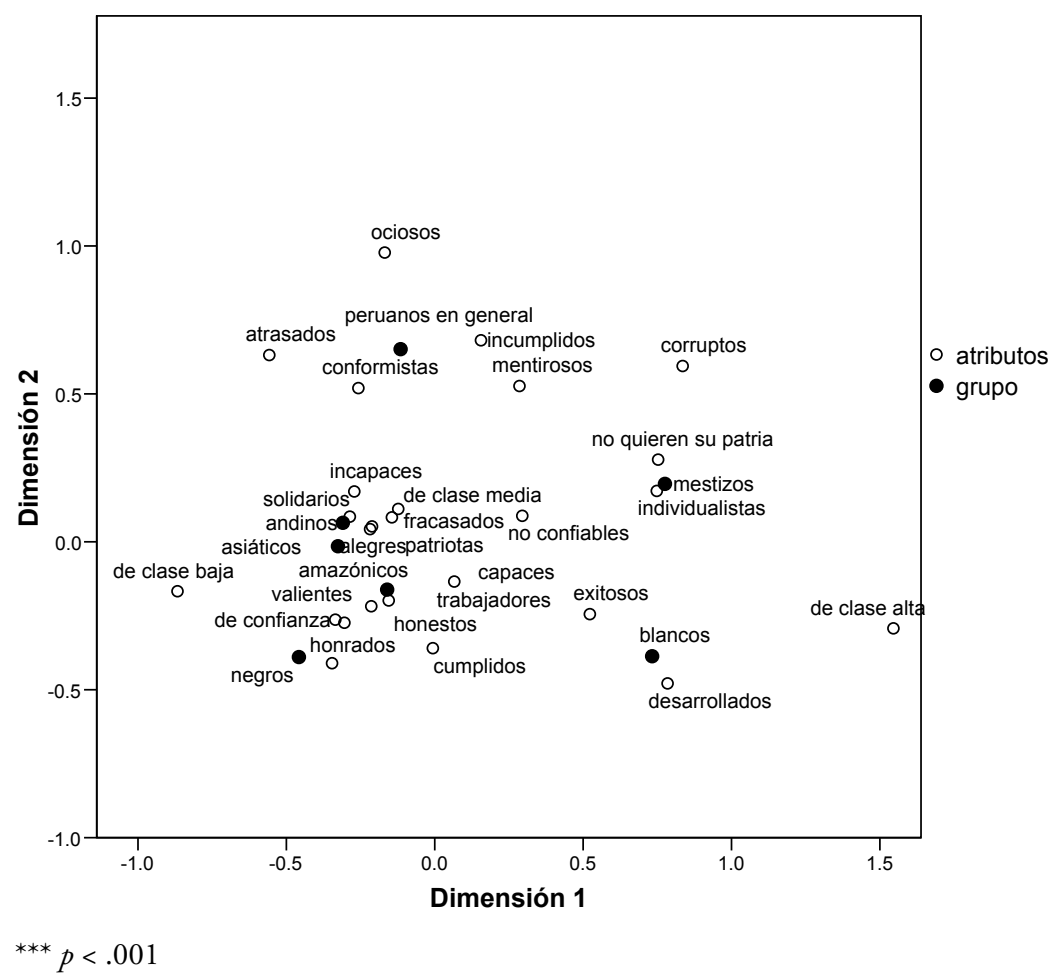

Figura 1. Análisis de correspondencias de las características estereotípicas asociadas a los grupos sociales evaluados. 


\section{Identificación con el Perú y con los grupos sociales evaluados}

En una escala del 1 al 5, la puntuación media en la identificación con la categoría social peruano de la muestra total fue de $3.23(D E=.79)$. Del mismo modo, la identificación con la categoría mestizo fue de 3.23 $(D E=1.02)$, seguido por la identificación con los amazónicos $(M=2.96$; $D E=1.17)$ y la identificación con los andinos $(M=2.94 ; D E=1.17)$. Por debajo de la media de estos últimos grupos, se ubica la identificación con los afroperuanos $(M=2.66 ; D E=1.09)$ y con los peruanos blancos $(M=2.53 ; D E=.94)$. Por último, se encontró que la puntuación media de la identificación con los asiáticos fue de $2.01(D E=1.13)$.

En la figura 2, se muestra la comparación de medias con la prueba $t$ de Student de las puntuaciones de identificación con los grupos étnicos en el caso de la muestra de nivel marginal y pobreza extrema con la muestra del estudio de Espinosa et al. (2007), constituida por personas de clase media y media alta de Lima Metropolitana $(n=81)$.

Como se observa, la identificación con los peruanos en general, mestizos, asiáticos y blancos es significativamente menor en la muestra de nivel marginal y pobreza extrema que en la de nivel medio y medio-alto. Sin embargo, lo contrario sucede en la identificación con los amazónicos, donde la media del nivel marginal es significativamente mayor que en el nivel medio y medio-alto. Esto no sucede en el caso de la identificación con los andinos y afroperuanos, ya que no se encuentran diferencias significativas cuando se comparan las medias de ambos niveles.

\section{Prejuicio individual y prejuicio percibido hacia los grupos sociales evaluados}

Con respecto al prejuicio individual, la valoración que los participantes hacen de los grupos étnicos sugiere que los grupos peor evaluados son los peruanos asiáticos $(M=5.42 ; D E=2.55)$, los afroperuanos $(M=5.81 ; D E=2.44)$ y los peruanos blancos $(M=6.01 ; D E=2.36)$. 
Representaciones estereotípicas y expresión del prejuicio en el Perú / Pancorbo, Espinosa y Cueto

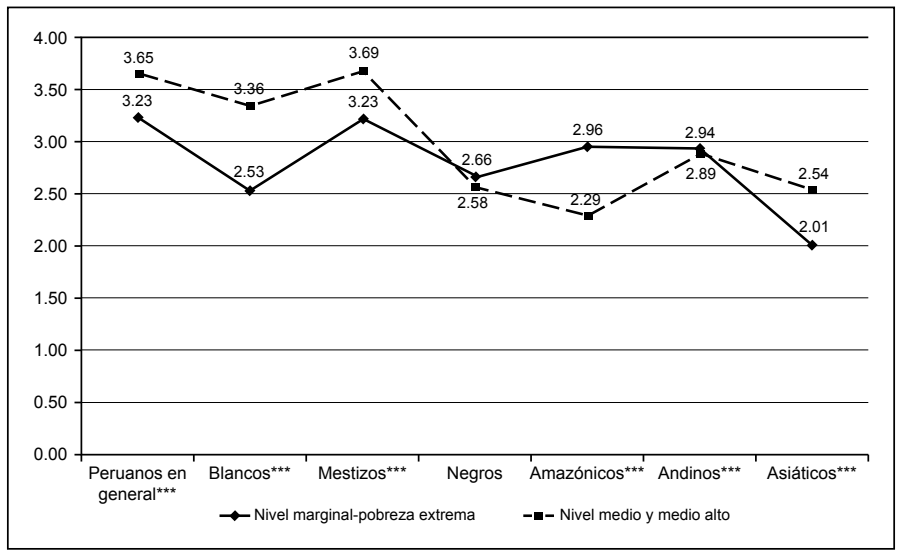

*** $p<.001$

Figura 2. Comparación de medias con la prueba $t$ de Student para muestras independientes, según identificación con los grupos étnicos por condición socioeconómica.

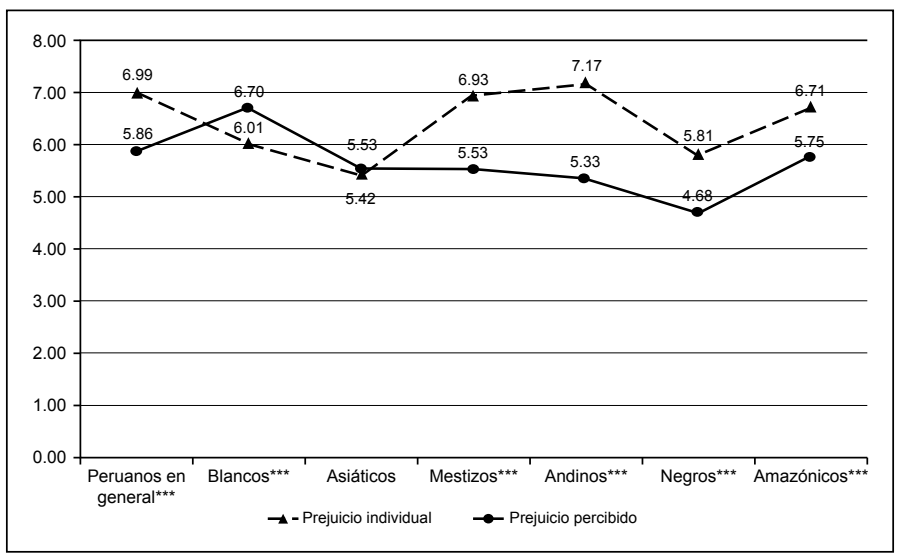

*** $p<.001$

Figura 3. Comparación de medias con la prueba $t$ de Student para muestras relacionadas, según el prejuicio individual versus el prejuicio percibido ante grupos étnicos. 
Por otro lado, los grupos mejor evaluados fueron los peruanos andinos $(M=7.17 ; D E=2.15)$, los peruanos en general $(M=6.99$; $D E=2.15)$, los peruanos mestizos $(M=6.93 ; D E=2.32)$ y amazónicos $(M=6.71 ; D E=2.53)$.

Así mismo, en la presente investigación, se pidió a los participantes que refirieran la valoración que haría un peruano promedio de los distintos grupos étnicos y se encontraron ciertas diferencias significativas en cuanto a la comparación de estos puntajes con los puntajes de la evaluación personal que los participantes hacían sobre los mismos grupos.

Como se puede observar, se encontraron puntajes significativamente más bajos en la evaluación atribuida a un peruano promedio sobre las categorías de peruanos en general, mestizos, andinos, amazónicos y afroperuanos, en comparación con la evaluación individual sobre estos grupos. Mientras tanto, solo en el caso de los peruanos blancos, el puntaje de la media de la evaluación percibida, o la evaluación que se piensa tiene el peruano promedio, fue mayor que la evaluación individual sobre este grupo. En el único caso en el que no se encontraron diferencias significativas fue en los puntajes de las medias de los peruanos asiáticos.

Por otro lado, la comparación a través de la prueba $t$ de Student, de las puntuaciones medias del prejuicio individual hacia los grupos étnicos en la muestra de nivel marginal y extrema pobreza y en la muestra de nivel medio y medio-alto, se muestra en la figura 4.

Se puede ver en la figura 4 que la evaluación de los participantes del nivel medio y medio-alto, de los peruanos mestizos, andinos y amazónicos es significativamente menor que la evaluación de los mismos grupos por parte de los participantes del nivel marginal y extrema pobreza. Por el contrario, la evaluación del grupo de asiáticos en el nivel marginal es significativamente menor que la evaluación del mismo grupo en el nivel medio y medio-alto. No se encontraron diferencias significativas entre evaluaciones de las categorías de peruanos en general, peruanos afroperuanos y peruanos blancos, siendo los puntajes de las medias muy parecidos en el caso de los dos niveles. 


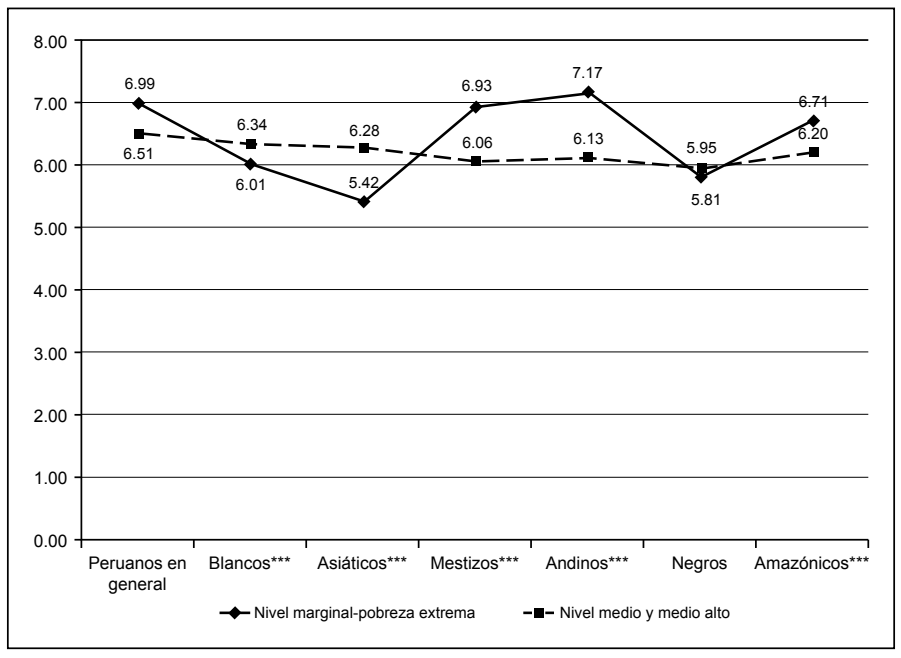

*** $p<.001$

Figura 4. Comparación de medias con la prueba $t$ de Student para muestras independientes, según el prejuicio individual hacia grupos étnicos por condición socioeconómica.

Ahora bien, se compararon los puntajes de las medias de la evaluación percibida del peruano promedio de los diferentes grupos en los dos niveles de estudio y se encontró que los participantes del nivel medio y medio-alto tienen puntajes significativamente más bajos con respecto a la evaluación percibida del peruano promedio sobre grupos andinos, amazónicos y afroperuanos que la evaluación percibida sobre estos mismos grupos en la muestra de nivel marginal y pobreza extrema. No se encontraron diferencias significativas en los puntajes de las medias en la evaluación percibida de los peruanos en general, mestizos, asiáticos y blancos. Estos resultados se muestran en la figura 5 . 


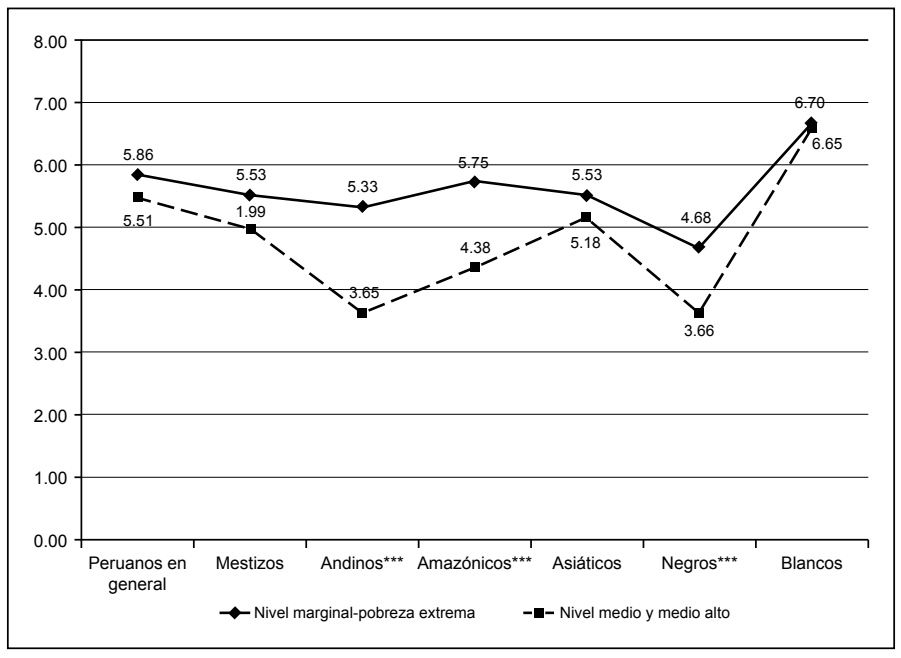

*** $p<.001$

Figura 5. Comparación de medias con la prueba $t$ de Student para muestras independientes, según el prejuicio percibido hacia grupos étnicos por condición socioeconómica.

\section{Identidad nacional peruana e identificación y valoración de grupos sociales}

Por otro lado, para el caso del presente estudio, se correlacionaron las respuestas de identificación general con el Perú con los niveles de identificación con cada grupo étnico, y la valoración de la categoría nacional con las valoraciones de estos grupos.

Acerca de la relación entre la identificación con el Perú y la identificación con los distintos grupos étnicos, se encontró que a mayor identificación con el Perú, mayor identificación con los blancos, $r$ (113) $=.39, p<.01$; con los mestizos, $r(115)=.24, p<.05$; $y$ con los afroperuanos, $r(115)=.24, p<.05$. 
Del mismo modo, se llevó a cabo una regresión lineal múltiple tomando a la identificación con el Perú como variable dependiente y la identificación con cada grupo étnico como variables independientes. La regresión resultó significativa, $F(6,115)=5.78, p<.01, R$ múltiple $=.49$ y explicó el $25 \%$ de la varianza. La beta significativa fue la de identificación con los peruanos blancos $(\beta$ estandarizada $=.416 ; p<$ $.001) \mathrm{y}$ con los peruanos mestizos ( $\beta$ estandarizada $=.218 ; p<.01$ ); con lo cual se confirma que estas dos variables son buenas predictoras de la identificación nacional.

Por otro lado, se encontró una relación positiva entre la valoración de peruanos en general y la valoración de todos los grupos étnicos considerados en el presente estudio. Esto es, a mayor valoración de los peruanos, mayor valoración de los blancos, $r(115)=.57, p<.01$; asiáticos, $r(115)=.28, p<.01$; mestizos, $r(115)=.47, p<.01$; andinos, $r(115)=.35, p<.01$; afroperuanos, $r(115)=.20, p<.05$; y amazónicos, $r(114)=.33, p<.01$.

En cuanto a la regresión lineal múltiple de la valoración de los peruanos en general, como variable dependiente, y la valoración con los grupos étnicos, como variables independientes, esta resultó significativa, $F(6,115)=29.20, p<.01, R$ múltiple $=.70$ y explicó el $49 \%$ de la varianza. La beta significativa fue la de valoración de los peruanos blancos $(\beta$ estandarizada $=.355 ; p<.01)$, de los peruanos mestizos $(\beta$ estandarizada $=.300 ; p<.01)$ y de los peruanos andinos $(\beta$ estandari$z a d a=.164 ; p<.05)$. Con ello, se puede afirmar que las valoraciones de los peruanos blancos, mestizos y andinos, son buenas predictoras de la valoración de los peruanos en general.

Por último, con respecto a la relación entre la identificación y valoración con cada grupo, se encontró que la valoración positiva hacia un grupo tiende a asociarse a la identificación con el mismo, en todos los casos: amazónicos, $r(114)=.52, p<.01$; afroperuanos, $r(115)=.51$, $p<.01$, andinos, $r(115)=.47, p<.01$; blancos, $r(113)=.35, p<.01$; mestizos, $r(115)=.33, p<.01$; peruanos en general, $r(115)=.27, p<$ $.01 \mathrm{y}$ asiáticos, $r(115)=.25, p<.01$. 


\section{Comentarios}

La Teoría de la Identidad Social postula que existe una relación positiva entre la identificación con el grupo y valoración positiva del mismo, y los resultados corroboran la presencia de esta relación en el caso de todas las categorías analizadas en el estudio. Estas relaciones son más intensas entre la identificación y valoración positiva de las categorías de amazónicos, andinos y mestizos, a las cuales se podría considerar los endogrupos de la muestra, debido a que son los grupos con quienes más se identifican los pobladores, acerca de quienes tienen estereotipos más positivos y a quienes más valoran, lo cual podrían delinear su favoritismo endogrupal. La preferencia por estos grupos estaría motivada por el deseo de los participantes de pertenecer a un grupo social y de ser aceptado por sus miembros, lo que conlleva un sentimiento positivo al que se denomina identidad social positiva (Tajfel, 1984).

Ahora bien, en la medida en que los participantes buscarían tener una identidad social positiva para mantener su autoestima, distinguirían a su grupo en ciertas dimensiones que los favorezcan, al compararse con otros grupos (Hogg \& Abrams, 2001), lo que los llevaría a evaluar a su propio grupo más positivamente que a los otros y a expresar sesgos endogrupales basados en estereotipos, prejuicios o comportamientos discriminatorios (Fiske, 1998; Hewstone, Rubin \& Hazel, 2002).

De acuerdo con los resultados del presente estudio, los grupos con los cuales hay una menor identificación y valoración son los asiáticos, afroperuanos y blancos; por lo cual, se podría considerar, que estos son los exogrupos para los participantes. Sin embargo, la imagen de los asiáticos y afroperuanos no es negativa del todo, lo que sí sucede con la imagen y valoración de los peruanos blancos, hacia quienes se adscriben estereotipos y prejuicios más negativos. Lo anterior se pudo observar con mayor claridad en la parte cualitativa del estudio que será incorporada en la presente discusión para validar los resultados de la parte cuantitativa. 


\section{Los peruanos, conformistas y envidiosos}

La primera parte de la investigación sugiere una identificación y valoración positiva hacia los peruanos en general, así como estereotipos positivos acerca de este grupo, lo que podría indicar que se ha construido una identidad social positiva asociada a la categoría. Sin embargo, los resultados de carácter cualitativo, dan cuenta de estereotipos negativos que catalogan a los peruanos como conformistas, egoístas y envidiosos; adjetivos parecidos a los encontrados por Espinosa et al. (2007) en su estudio.

Los estereotipos asociados a la categoría nacional por los participantes en la parte cualitativa del presente estudio, se relacionarían con sus dificultades para alcanzar el progreso, que se percibe como una competición entre peruanos, caracterizada por la desunión, el egoísmo, la envidia y la pelea por los recursos.

"Te ven surgiendo a ti y piensan que estás haciendo algo malo, de dónde has sacado la plata, son mal pensados" (Grupo Focal 2)

"Solamente ellos [los peruanos] quieren para ellos mismos y no para los demás” (Grupo Focal 2)

En ese sentido, el estereotipo del peruano podría enmarcarse en una percepción negativa de la realidad nacional, que se relaciona con la realidad en la que viven los participantes. De este modo, dichos estereotipos se convertirían en mecanismos explicativos de cómo y porqué surgen dificultades de interacción social en este contexto, con lo cual cumplirían la función de racionalizar y justificar las condiciones en las que se estructura la sociedad (Yzerbyt et al., 1997).

\section{Los andinos y su cambio de lo rural a lo urbano}

La construcción del estereotipo de los andinos, al igual que la mayor identificación y valoración de los mismos, se relacionaría con la cercanía de los participantes a estos grupos y al contexto en el que viven, ya que una gran parte de ellos nació en la sierra, o tienen familiares o amigos que provienen de dicha región. 
Así, a los andinos se les asocia con estereotipos positivos como valientes, patriotas y solidarios, así como trabajadores, esforzados y progresistas.

"Lo que yo he vivido es que [los andinos] son muy buenas personas, muy educados, si es posible te dan su cama y ellos duermen en el suelo, son buenos anfitriones” (Grupo Focal 3)

"En realidad, [los andinos] son personas cultas que se saben valorar por sí mismas, que saben sobresalir y poner todo el esfuerzo y empeño para poder sobrevivir, sin el apoyo de nadie (...)" (Grupo Focal 4)

Así mismo, consideran que la bondad que caracteriza al andino se pierde al migrar a la ciudad donde se corrompe y se convierte en un ser desconfiado. Esto podría reflejar la visión de los participantes acerca del mundo urbano en contraposición al mundo rural, este último dotado de armonía, unión y riqueza de recursos, y aquel caracterizado por la delincuencia, la deshonestidad y el individualismo (Ortiz \& Yamamoto, 1999).

“- ¿Qué pasa cuando vienen a Lima? - Se malean, se corrompen” (Grupo Focal 3)

“- ¿Y en Lima dejan de ser unidos? - Sí, cada uno baila con su pañuelo” (Grupo Focal 3)

Por otro lado, en la etapa cualitativa de la investigación, los participantes afirmaron que perciben a los andinos como temerosos, callados, tímidos y poco alegres, lo que se evidencia cuando estos llegan a la ciudad y sienten desconfianza hacia personas que no son de su mismo lugar de procedencia, o cuando reflejan cierto temor a ser engañados por terceros. Además, esta percepción de tristeza y de emociones negativas en los andinos, podría atribuirse a un clima emocional de tristeza producto de sus condiciones de vida y del conflicto armado interno que sufrió mayoritariamente la sierra peruana en décadas pasadas (Theidon, 2009). 
Al igual que en la parte cualitativa de este estudio, en el estudio de Espinosa et al. (2007) con una muestra de nivel medio y medio-alto, se encuentra que los andinos son asociados a la tristeza y al mayor control emocional, así como a la honradez y el trabajo. Sin embargo, al mismo tiempo se les atribuye el atraso, el conformismo y el fracaso, atributos negativos que, de acuerdo con los autores, los alejan del desarrollo y el poder. Dichos atributos negativos son congruentes con la menor valoración de los andinos por parte de la muestra de personas de clase media y media alta, en comparación con los estereotipos positivos y la mejor valoración de la muestra de la presente investigación.

Adicionalmente, en el presente estudio y en el estudio de Espinosa et al. (2007) se asocia a los andinos con la solidaridad, cualidad que caracteriza a culturas colectivistas por la lealtad y dependencia entre sus integrantes (Gudykunst \& Ting-Toomey, como se citó en Gudykunst \& Bond, 1997).

Esto es, la característica de solidaridad adscrita a los andinos se podría relacionar a la percepción de que la cultura de este grupo se caracteriza por el colectivismo, y a la creencia de que la solidaridad es una forma de afrontar y adaptarse a las necesidades económicas y sociales de la realidad en la que viven.

\section{Los amazónicos, alegres y poco desarrollados}

De manera similar al caso de los andinos, en la presente investigación, existe una mayor identificación, valoración y estereotipos positivos asociados a los amazónicos, con quienes también hay mayor familiaridad y cercanía, debido a que una gran mayoría de los participantes procede de dicha región del país.

Sus estereotipos se asocian a la valentía y al patriotismo, referidos a la defensa de sus recursos naturales, así como al trabajo, la solidaridad y la alegría. Este último atributo es especialmente importante la percepción sobre los amazónicos y su cultura, la que es valorada por la diversidad de su música, bailes y comidas. Lo anterior se hizo evidente 
en las referencias acerca del estilo de vida en la Amazonía en la parte cualitativa de la investigación.

Los estereotipos asociados a los amazónicos se relacionan con lo descrito por Ortiz (2001) respecto a los mitos alrededor de la Amazonía, uno de los cuales afirma que este territorio está cargado de riquezas y recursos naturales que pueden parecer inagotables.

$\mathrm{Al}$ igual que en la presente investigación, los resultados de Espinosa et al. (2007) distinguen a los amazónicos por su alegría y solidaridad, y añaden que existe pobreza y poco desarrollo en esta región del país. En el estudio de Espinosa et al. (2007) también se asocia a los amazónicos a atributos como el atraso, la ociosidad y el conformismo. Dichos estereotipos se relacionan con otros mitos descritos por Ortiz (2001) sobre la pobreza que existe la Amazonía y sobre los nativos como un freno para el progreso.

\section{Los mestizos y el mestizaje como una representación social difícil de precisar}

Los resultados cuantitativos refieren que los participantes asociaron a la categoría mestizos atributos tales como el éxito, ser de clase alta, la capacidad y el desarrollo. Sin embargo, los participantes de la fase cualitativa no tuvieron claridad en describir cómo era este grupo, y tan solo alcanzaron a caracterizarlos como una mezcla de razas de diferente procedencia, usualmente asociados a las personas de tez oscura, como se puede observar en las citas a continuación.

"Es un poco difícil de explicarlo porque ese término se usaba para otra época” (Grupo Focal 2)

"Personas que alguna manera son un poquito marginadas, de color, que no tienen una identidad propia, más o menos, son una mezcla de diferentes razas" (Grupo Focal 1)

A pesar de la poca claridad en la definición acerca de lo que significa ser mestizo, este es el grupo étnico con quién más se identifican los participantes en la parte cuantitativa del estudio. Además, junto con 
los andinos y amazónicos, la categoría mestizos es la que se asocia más intensamente a la identificación y valoración de la categoría peruano; lo que abre la interrogante acerca de la poca claridad de sus contenidos estereotípicos y su definición, lo que en cierta medida también podría estar asociado a la poca claridad con que se define la categoría nacional.

Al definir a la categoría como una mezcla de razas, el estereotipo del mestizo puede haberse construido a partir de la idea de diversidad étnica; esto es, no como una categoría a la que se asocia una territorialidad o cultura específica, sino como una integración de diversas culturas, razas y pensamientos, por lo cual podría entenderse la dificultad para delimitarla o distinguirla de las demás categorías.

\section{Los afroperuanos, poco honrados y discriminados}

Los afroperuanos son uno de los grupos con quien menos se identifican y a quienes menos valoran los participantes del estudio. Son catalogados como trabajadores, valientes, alegres y patriotas, pero también como "choros" (ladrones), "lisurientos" y poco honrados. Describen que es un grupo que impresiona por su aspecto, su color y su manera de comportarse, ya que hablan fuerte, lo que llama la atención de la gente.

"Tú conoces a un negro por el vocabulario que tienen, son más vozarrones, te alzan la voz, te hablan más fuerte” (Grupo Focal 4)

Así mismo, los participantes consideran que son el grupo que menos valora y más discrimina la sociedad peruana, por considerarlos delincuentes y deshonestos.

"Yo de chiquilla, me crié por la avenida Argentina [...] y nosotros éramos los únicos claritos, toditos eran negritos. Yo he vivido ahí, pero ha sido bien peligroso porque consumen droga, son choros, todos malcriados. [...] A mí me daba miedo salir de mi casa" (Grupo Focal 3) 
En el estudio de Espinosa et al. (2007) se encuentran igualmente estereotipos negativos, una menor identificación y una menor valoración hacia este grupo. La muestra del presente estudio los cataloga como alegres, conformistas, no confiables, ociosos y atrasados; y admite, igualmente, que es uno de los grupos a quien menos valora la sociedad peruana.

Relativo a lo anterior, Benavides, Torero y Valdivia (2006), en un estudio sobre los afrodescendientes en el Perú, hallan en un primer estudio cuantitativo que una reducida proporción de personas afroperuanas reporta haberse sentido objeto de discriminación o haber recibido algún tipo de trato discriminatorio. Sin embargo, esta misma población, en un segundo estudio cualitativo, admite que la discriminación es predominante hacia los afroperuanos, a quienes se les asocia con el robo, la delincuencia y la criminalidad.

Los autores atribuyen esta diferencia a la resistencia de sus encuestados a reconocer la discriminación de la que han sido objeto. A su vez, llegan a la conclusión de que existe una cierta ambivalencia en la identidad de este grupo: si bien se reivindica y aparece el "orgullo negro" en algunos casos, en otros existe el deseo de "blanqueamiento", lo que podría deberse al rechazo de su condición racial "negra", a contracorriente de la afirmación de su identidad. Tal como afirman los autores "por un lado, su identificación está basada fuertemente en la raza y, por otro, los procesos de mestizaje a los que dicha población está expuesta socavarían la base de dicha identificación" (Benavides et al., 2006, p. 10).

La discriminación a la que hace alusión el estudio de Benavides et al. (2006) es congruente con lo observado en los participantes del nivel marginal y de extrema pobreza del presente estudio, así como los participantes del estudio con muestras del nivel socioeconómico medio y medio-alto, acerca de cómo los afroperuanos son discriminados por el resto de la sociedad.

En ambas muestras, la valoración individual hacia los afroperuanos es más alta que la valoración que ellos perciben tienen los otros peruanos de este grupo, lo que también ocurre con los otros grupos evaluados en el estudio, salvo el caso de los blancos. Esto se explicaría en la lógica de "los otros son más prejuiciosos que yo". Según Espinosa et al. (2007) la 
brecha entre la valoración personal y la atribuida al peruano promedio sugiere que en el contexto peruano nadie se considera prejuicioso, ya que serlo resultaría políticamente incorrecto. Esto iría de acuerdo con el concepto de racismo aversivo, que afirma que hay ciertas creencias y sentimientos que la persona trata de disociar de la imagen no-prejuiciosa que desea mostrar (Fiske \& Taylor, 2007).

Especialmente, para la muestra del presente estudio, podría estarse evidenciando un racismo de tipo aversivo hacia los afroperuanos, ya que se hacen explícitos estereotipos negativos hacia este grupo, pero se combinan con una simpatía paternalista, en tanto se les caracteriza como alegres y buenas personas. Lo anterior a pesar de una no identificación con este grupo y una muy baja valoración del mismo. En concordancia con lo anterior, las actitudes negativas hacia este grupo podrían manifestarse en comportamientos no intencionales, cuando estos no sean atribuidos a algún aspecto racial (Dovidio, 2001).

\section{Los asiáticos, trabajadores y emprendedores}

En el presente estudio el estereotipo de los asiáticos se asocia al trabajo, la valentía, la solidaridad, el empeño, el progreso y la riqueza. Adicionalmente, son considerados "secos" y reservados, poco honrados, así como no integrados con el resto de los grupos del país.

“- A mi parecer son personas secas, que no me atraen mucho la atención.

- ¿Cómo secos?

- Por la actitud que ellos tienen, no son expresivos, son más serios, más reservados" (Grupo Focal 4)

Por su parte, en el estudio de Espinosa et al. (2007) la muestra del nivel medio y medio-alto describe a los asiáticos como trabajadores, cumplidos, capaces, honrados y exitosos, y al contrario de lo visto en el nivel marginal y de extrema pobreza, este es el segundo grupo étnico más valorado por los participantes de clase media, solo por debajo de los peruanos blancos. De acuerdo con Espinosa et al. (2007), los asiáticos 
comparten con los blancos características asociadas al poder y se les incluye entre los grupos de mayor estatus en la sociedad peruana.

Siguiendo el modelo de estereotipia de Fiske, Cuddy, Xu y Glick (2002), los estereotipos de los asiáticos en los participantes de nivel marginal y extrema pobreza se relacionarían con una alta competencia (progreso, capacidad y riqueza) y baja calidez (personas "secas" y reservadas). Según el modelo, este tipo de estereotipos incentivan prejuicios como la envidia y el resentimiento en quienes los perciben. Coincidentemente, los estudios de Fiske et al. (2002) encuentran que estos estereotipos y prejuicios tienden a orientarse hacia asiáticos, judíos y personas ricas en contextos distintos al peruano.

\section{Los blancos, ricos, exitosos y poderosos}

Por un lado, en la etapa cuantitativa del estudio se apreció que los blancos son percibidos como trabajadores, desarrollados, exitosos, capaces y cumplidos. Sin embargo, los resultados cualitativos de la investigación relativizan algunos de estos atributos.

$\mathrm{Al}$ respecto, los participantes del grupo de nivel marginal y extrema pobreza reconocen a los blancos como capaces, aunque esto lo atribuyen al hecho de tener recursos económicos para capacitarse. También se les considera trabajadores, pero no de la misma forma que los andinos, $\mathrm{y}$ es que a los blancos los distingue el hecho de tener plata y tener personas de menor estatus a su cargo, quienes son los que ponen el mayor esfuerzo en sus empresas y a quienes muchas veces esclavizan con malos pagos o maltrato.

"Trabajadores puede ser a punto de sus capacidades, inteligencia, pero el que hace el esfuerzo es la gente humilde, los que trabajan para ellos" (Grupo Focal 4)

"Ellos piensan que con el dinero pueden comprar cualquier cosa, pero si ellos pueden tener dinero, es gracias a los provincianos que vienen acá a ofrecer su trabajo y ellos se aprovechan de eso y exigen trabajar y lo que van a pagar es una mínima porción, y aún así esclavizan" (Grupo Focal 4) 
Del mismo modo, los participantes afirmaron que la mayoría de blancos discrimina a las personas más pobres por no tener su misma condición socioeconómica, aunque no consideran que sea este grupo el único que discrimina sino, en general, la clase adinerada en el Perú. Con ello, se puede ver que se atribuye la discriminación a una cuestión económica más que racial.

"Te marginan, porque no eres de su mismo nivel social" (Grupo Focal 1)

- [El racismo sucede] porque tienen plata, es porque son blancos y porque nosotros somos pobres pues" (Grupo Focal 3)

Los estereotipos y prejuicios acerca de los blancos se enmarcan en la visión de que hay exclusión e inequidad en la realidad peruana. Afirman que es el grupo con mejor condición socioeconómica y que sus miembros tienen acceso a una mejor educación, a mejores oportunidades de trabajo y obtienen mejores servicios por parte del Estado, en contraposición a sectores más humildes como los andinos, amazónicos $\mathrm{y}$ afroperuanos.

De acuerdo con lo señalado en la revisión teórica, las personas integrarían la información del medio para formar una estructura de la sociedad coherente, donde se ubican el grupo discriminador y el grupo discriminado, en este caso, el grupo de blancos adinerados, frente a los grupos más humildes, sector al que los participantes se adscriben. Así, sus estereotipos les permitirían explicar y racionalizar por qué se da esta situación (Yzerbyt et al., 1997), y estos junto con sus prejuicios les ayudarían a preservar el sentimiento positivo relativo a su identidad social (Tajfel, 1984).

Contrario a este caso, la muestra del estudio de Espinosa et al. (2007) se identifica y valora más a los blancos, es decir, al grupo de los discriminadores, más que a los otros grupos, y considera que este grupo es desarrollado, exitoso y capaz, pero individualista y corrupto.

De acuerdo con Espinosa et al. (2007) el desarrollo, el éxito y la capacidad serían atributos bien valorados por los participantes, mientras 
que la corrupción no sería un atributo negativo, ya que es visto como un recurso para legitimar o mantener el poder. Estos resultados soportan la idea de que ser blanco en el Perú asigna alto estatus y prestigio (Espinosa et al., 2007; Portocarrero, 1992).

Conforme al modelo de estereotipia de Fiske et al. (2002), los estereotipos de los blancos asignados por los participantes del nivel marginal y pobreza extrema se verían caracterizados por una alta competencia (capaces y exitosos), pero una baja calidez (presumidos y discriminadores). Una vez más, según este modelo, estos estereotipos se acompañarían por prejuicios como la envidia y el resentimiento, los que usualmente suscitan exogrupos socioeconómicamente exitosos, que son percibidos como una amenaza para el endogrupo.

En cambio, los estereotipos asignados a los blancos por el nivel medio y medio-alto se caracterizarían por una alta competencia (desarrollados y exitosos) y una alta calidez (alta valoración de este grupo), y se verían acompañados por actitudes de admiración y orgullo hacia este grupo (Espinosa et al., 2007). Según Fiske et al. (2002), usualmente estos estereotipos y actitudes son asociados al endogrupo.

Por su parte, el estudio de Portocarrero (1993) parece apoyar parcialmente los resultados del presente estudio, ya que encuentra que, en los sectores urbano-marginales y pobres, lo blanco es asociado con la riqueza, el poder y la felicidad, lo que se ve acompańado por emociones tanto de admiración como de odio y resentimiento. Las diferencias sociales no parecen cuestionarse y la agresividad se expresa más hacia la categoría blanco que hacia la de rico, por lo que concluye, existe una afectividad hostil hacia lo blanco.

En el presente estudio, en cambio, las diferencias sociales, tanto como la inequidad que perciben los participantes, se cuestionan, y los sentimientos negativos como la agresividad parecen asociarse al blanco por ser rico, y no únicamente por ser blanco.

$\mathrm{Al}$ respecto, se reconoce que detrás del discurso de los participantes existe resentimiento y cierto odio hacia los grupos que tienen mayor poder y estatus en el país, donde se ubican principalmente los blancos. Sin embargo, no se pudo distinguir con claridad qué papel cumple el 
aspecto racial, el económico o el cultural como mediadores de dichos sentimientos intergrupales.

La poca claridad al distinguir el papel de la raza como marcador de diferencia en las relaciones intergrupales de los participantes se podría ver influenciado por la culpa y vergüenza que, usualmente, dificultan el aceptar que existe racismo en el Perú (Portocarrero, 1992).

Lo expuesto por Portocarrero (1992) parece apoyar la clara percepción de los participantes de que en la sociedad peruana existen jerarquías y brechas que dividen a unos grupos con mayor estatus y poder de otros que no gozan de las mismas oportunidades (andinos, amazónicos y afroperuanos) y que son desvalorizados por el resto de peruanos. Es bajo este marco de desigualdad y exclusión, que se expresan los estereotipos y prejuicios en la sociedad peruana.

El peligro de la desigualdad y la exclusión radica en la manifestación del odio y el resentimiento de unos grupos frente a otros, lo que deriva en el conflicto y la violencia (Tajfel \& Turner, 1979/2001), tal como aconteció en la década de los años ochenta y noventa con el mayor conflicto interno vivido en el país, cuyas principales víctimas fueron los indígenas. En ese sentido, la Comisión de la Verdad y la Reconciliación (2008) afirma que "de cada cuatro víctimas, tres fueron campesinos o campesinas cuya lengua materna era el quechua. Se trata de un sector de la población históricamente ignorado por el Estado y por la sociedad urbana, aquella que sí disfruta de los beneficios de nuestra comunidad política” (p. 10).

Así, el presente estudio no solo describe los estereotipos y prejuicios hacia grupos étnicos que tienen los participantes, sino que a su vez, logra distinguir cómo los participantes perciben ciertos comportamientos discriminatorios que tienen los grupos de mayor estatus y poder frente a los grupos de menor condición socioeconómica. Dichos patrones de relación intergrupal, se ubican en un contexto de exclusión social e inequidad existentes en la sociedad peruana, desde la perspectiva de los participantes. Este marco acentúa las diferencias entre los grupos y justifica la aparición de los estereotipos y prejuicios que se manifiestan en los resultados de la presente investigación. 


\section{Referencias}

Allport, G. (1954). The nature of prejudice. Cambridge, MA: AddisonWesley.

Asociación Peruana de Empresas de Investigación de Mercados, APEIM. (2010). Niveles socio-económicos 2010. Lima Metropolitana. Recuperado de http://www.apeim.com.pe/niveles.html

Benavides, M., Torero, M. \& Valdivia, N. (2006). Más allá de los promedios: afro descendientes en América Latina: pobreza, discriminación social e identidad: el caso de la población afrodescendiente en el Perú. Washington, DC: World Bank.

Bustamante, R. (1986). Raza e identidad social positiva y negativa en Lima. En F. León (Ed.), Psicología y realidad peruana: el aporte objetivo. Lima: Mosca Azul.

Comisión de la Verdad y Reconciliación. (2008). Hatun Willakuy: versión abreviada del informe final de la Comisión de la Verdad y Reconciliación. Lima: Autor.

Dovidio, J. (2001). On the nature of contemporary prejudice: The third wave. Journal of Social Issues, 5, 829-849.

Dovidio, J. \& Gaertner, S. (1999). Reducing prejudice: Combating intergroup biases. Current Directions in Psychological Science, 8, 101-109.

Espinosa, A. (2003). Identidad social e identidad nacional en una muestra de triciclistas en Juliaca (Tesis de licenciatura inédita). Pontificia Universidad Católica del Perú, Lima.

Espinosa, A. (2011). Estudios sobre identidad nacional en el Perú y sus correlatos psicológicos, sociales y culturales (Tesis doctoral inédita). Universidad del País Vasco, San Sebastián, España.

Espinosa, A., Calderón-Prada, A., Burga, G. \& Guímac, J. (2007). Estereotipos, prejuicios y exclusión social en un país multiétnico: el caso peruano. Revista de Psicología, 25(2), 295-338.

Fiske, S. (1998). Stereotyping, prejudice and discrimination. En D. Gilbert, S. Fiske \& G. Lindzey (Eds.), Handbook of social psychology (pp. 357-412). Boston: McGraw-Hill. 
Fiske, S., Cuddy, A., Xu, J. \& Glick, P. (2002). A model of (often mixed) stereotype content: Competence and warmth respectively follow from perceived status and competition. Journal of Personality and Social Psyhology, 82, 878-902.

Fiske, S. \& Taylor, S. (2007). Social cognition: From brains to culture. Nueva York: McGraw- Hill.

Gaertner, S. L. \& Dovidio, J. F. (1986). The aversive form of racism. En J. F. Dovidio \& S. L. Gaertner (Eds.), Prejudice, discrimination and racism (pp. 61-90). San Diego: Academic Press.

Gudykunst, W. B. \& Bond, M. H. (1997). Intergroup relations across cultures. En J. W. Berry, M. H. Segall \& C. Kagitcibasi (Eds.), Handbook of cross-cultural psychology (pp. 119-161). Boston: Allyn \& Bacon.

Hewstone, M., Rubin, M. \& Hazel, W. (2002). Intergroup bias. Annual Review of Psychology, 53, 575-604.

Hogg, M. A. \& Abrams, D. (2001). Intergroup relations: An overview. En M. A. Hogg \& D. Abrams (Eds.), Intergroup relations: Key readings in social psychology (pp. 1-14). Filadelfia: Psychology Press.

McGarty, C., Yzerbyt, V. \& Spears, R. (2002). Social, cultural and cognitive factors in stereotype formation. En C. McGarty, V. Yzerbyt \& R. Spears (Eds.), Stereotypes as explanations: The formation of meaningful beliefs about social groups (pp. 1-15). Cambridge: Cambridge University Press.

Moghaddam, F. M. (1998). Social psychology: Exploring universals across cultures. Nueva York: W. H. Freeman and Company.

Moghaddam, F. M., Taylor, D. M. \& Wright, S. C. (1993). Social psychology in cross-cultural perspective. Nueva York: W. H. Freeman and Company.

Ortiz, A. (2001). Manual de etnografía amazónica. Lima: Fondo Editorial de la Pontificia Universidad Católica del Perú.

Ortiz, A. \& Yamamoto, J. (1998). Mixturas. Anthropologica, 17, 225244. 
Portocarrero, G. (1992). Del racismo al mestizaje: una apuesta por la integración. En Centro de Investigación Cerámica (Ed.), Los quinientos años: sin espacio para la reflexión (pp. 31-41). Lima: Allpamérica.

Portocarrero, G. (1993). La cuestión racial: espejismo y realidad. En G. Portocarrero (Ed.), Racismo y mestizaje. Lima: SUR Casa de Estudios del Socialismo.

Stangor, C. (2000). Overview. En C. Stangor (Ed.), Stereotypes and prejudices (pp. 1-16). Ann Arbor, MI: Edwards Brothers.

Tajfel, H. (1984). Grupos humanos y categorias sociales. Barcelona: Herder. Tajfel, H. \& Forgas, J. (1981). Social categorization: Cognitions, values and groups. En C. Stangor (Ed.), Stereotypes and prejudice (pp. 49-63). Ann Arbor, MI: Edwards Brothers.

Tajfel, H. \& Turner, J. (1979/2001). An integrative theory of intergroup conflict. En M. A. Hogg \& D. Abrams (Eds.), Intergroup relations: Key readings in social psychology (pp. 94-109). Filadelfia: Psychology Press.

Theidon, K. (2009). Entre prójimos: el conflicto armado interno y la politica de reconciliación en el Perú. Lima: IEP.

Yzerbyt, V., Rocher, S. J. \& Schadron, G. (1997). Stereotypes as explanations: A subjective essentialistic view of group perception. En R. Spears, P. J. Oakes, N. Ellemers \& S. A. Haslam (Eds.), The social psychology of stereotyping and group life (pp. 20-50). Oxford: Blackwell.

Zárate, M. (2009). Racism in the $21^{\text {st }}$ Century. En T. Nelson (Ed.), Handbook of prejudice, stereotyping, and discrimination (pp. 387406). Nueva York: Psychology Press.

Recibido: 15 de junio, 2011 Aceptado: 15 de julio, 2011 